\title{
Модификация смачиваемости углеродных нанотрубок с помощью ионного облучения
}

\author{
(C) А.И. Морковкин ${ }^{1}$, Е.А. Воробьева ${ }^{2}$, А.П. Евсеев ${ }^{1,2}$, Ю.В. Балакшин ${ }^{2,3}$, А.А. Шемухин ${ }^{2,3}$ \\ ${ }^{1}$ Московский государственный университет им. М.В. Ломоносова (физический фракультет), \\ 119991 Москва, Россия \\ ${ }^{2}$ Московский государственный университет им. М.В. Ломоносова, НИИ ядерной физики им. Д.В. Скобельцына, \\ 119991 Москва, Россия \\ ${ }^{3}$ Центр квантовых технологий Московского государственного университета им. М.В. Ломоносова, \\ 119991 Москва, Россия \\ E-mail: a.i.morkovkin@physics.msu.ru
}

Поступила в Редакцию 8 июля 2019 г.

В окончательной редакции 15 июля 2019 г.

Принята к публикации 15 июля 2019 г.

\begin{abstract}
Приведены результаты исследования изменения смачиваемости коммерческих многостенных углеродных нанотрубок (МУНТ) Таунит-МД в результате облучения ионами $\mathrm{Ar}^{+}$с энергией 120 кэВ различными флюенсами. При помощи спектроскопии комбинационного рассеяния света и сканирующей электронной микроскопии исследована структура облученных МУНТ, и проведен рентгеноспектральный микроанализ образцов. Рассмотрены зависимости среднего диаметра МУНТ, концентрации $\mathrm{O}_{2}$ и дефектности МУНТ в образцах от флюенса облучения, а также их влияние на угол смачиваемости дистиллированной водой, этиленгликолем и циклогексаном. Обсуждаются возможность и перспективы использования ионно-пучковых методов модификации для контролируемого изменения угла смачиваемости с целью создания гидрофобного или гидрофильного к различным видам жидкостей покрытия из МУНТ.
\end{abstract}

Ключевые слова: ионное облучение, многостенные углеродные нанотрубки, сканирующая электронная микроскопия, комбинационное рассеяние света, смачиваемость, метод сидячей капли.

DOI: 10.21883/FTP.2019.12.48629.9207

\section{1. Введение}

Уникальные физические и химические свойства углеродных нанотрубок (УНТ) вызывают интерес исследователей с момента их открытия в 1991 г. С. Иидзимой [1]. Особенности УНТ инициируют непрерывные поиски возможных применений их уникальных характеристик для улучшения современных технологических процессов [2-8]. Одним из актуальных направлений стало исследование характера взаимодействия УНТ с различными жидкостями.

Многие исследования показывают [9-11], что синтезированные многостенные углеродные нанотрубки (МУНТ) проявляют гидрофобный характер (в некоторых случаях они проявляют супергидрофобный характер - угол смачивания от 150 до 180) [12]; также встречаются данные о том, что одностенные углеродные нанотрубки проявляют гидрофобный или гидрофильный характер в зависимости от их среднего диаметра [13].

Управление смачиваемостью углеродных нанотрубок вплоть до получения супергидрофобности востребовано для создания устройств микрофлюидики типа „лаборатория на чипе“ (lab-on-a-chip) [14]. Гидрофобные УНТ предлагают использовать для увеличения емкости современных аккумуляторов (например, литий-ионных батарей [15]) или создания суперконденсаторов [16], которые могут заменить в будущем привычные нам элементы питания.
Гидрофильные УНТ имеют потенциал к использованию в области водородной энергетики для безопасного хранения водорода [17] или в качестве элементов высокочувствительных датчиков влажности [18].

Согласно ряду исследований, модификация УНТ после их синтеза позволяет изменять характер взаимодействия УНТ с различными жидкостями. Например, в работе [19] наблюдается обратимый эффект изменения характера взаимодействия МУНТ с водой с гидрофобного на гидрофильный после облучения ультрафиолетовым излучением УНТ-покрытия, что связано с образованием радикалов, и затем обратно на гидрофобный после выдержки покрытия в темной комнате.

Благодаря особенностям технологии ионной имплантации разработка методики контролируемого изменения угла смачиваемости поверхности массива УНТ различными жидкостями представляет практический интерес. Ионное облучение является одним из методов варьирования свойств УНТ после синтеза, что дает возможность контролируемого изменения параметров УНТ путем использования определенных режимов облучения [20,21]. Авторы работы [10] показали, что облучение ионами $\mathrm{Na}^{+}$многостенных углеродных нанотрубок позволяет изменять их изначально супергидрофобный характер на гидрофильный. Однако аспекты влияния ионных пучков на характер взаимодействия МУНТ с различными жидкостями представляют интерес в связи с малым количеством публикаций по данной тематике. 
С целью изучения возможностей применения ионного облучения для изменения смачиваемости МУНТ были проведены экспериментальные работы по облучению коммерческих МУНТ Таунит-МД.

\section{2. Экспериментальные методы}

Образцы были изготовлены из коммерческих МУНТ Таунит-МД (ТМД) путем прессовки в цилиндрической форме при давлении в 4 т (метрические тонны). Таунит-МД представляют собой неориентированные МУНТ с диаметром $d_{\mathrm{CNT}} \approx 17-23$ нм. Образцы имели размеры $10 \times 2$ мм в форме цилиндров с массой $m \approx(60 \pm 2)$ мг.

Ионное облучение проводилось под углом $7^{\circ}$ к нормали к поверхности образца ионами $\mathrm{Ar}^{+} \mathrm{c}$ энергией 120 кэВ тремя флюенсами, $10^{12}, 4 \cdot 10^{14}, 2 \cdot 10^{15} \mathrm{~cm}^{-2}$ на ускорителе HVEE-500 [22-24]. Однородность облучения достигалась сканированием с большой частотой поверхности образца пучком в двух взаимно перпендикулярных направлениях.

Для исследования структуры МУНТ после ионного облучения были сняты изображения в сканирующем электронном микроскопе (СЭМ) Carl Zeiss Ultra 55 SEM и проведен рентгеноспектральный микроанализ (РСMА) поверхности образца.

Также были сняты спектры комбинационного рассеяния света (КРС) на приборе ЗНЛ ИНТЕГРА Спектра с использованием лазера с длиной волны $\lambda=473 \mathrm{нм.}$ Сглаживание спектров осуществлялось с помощью частотной фурье-фильтрации. Все спектры аппроксимировались комбинацией лоренцианов и гауссианов. Для расчета отношения интенсивности $D$ - и $G$-пиков, $I_{D} / I_{G}$, использовались значения интегрированной площади под аппроксимирующими пиками.

Измерение угла смачиваемости проводилось методом сидячей капли при следующих условиях: давление $P=1$ атм, комнатная температура. Для проведения измерений был использован набор из трех жидкостей различной полярности: с большой полярностью - дистиллированная вода, со средней полярностью - этиленгликоль, неполярный циклогексан. Жидкости наносились на образец при помощи механической пипетки.

Угол смачиваемости определялся путем анализа профиля капли на макрофото, снятого в момент нанесения капли на поверхность образца. Процедура анализа включает в себя следующие этапы: по форме капли вписывается окружность некоторого радиуса, далее эта окружность описывается квадратом, в котором затем строятся диагонали для нахождения точного расположения центра вписанной окружности; затем на осветленном и контрастном фото проводится поиск границы раздела между каплей и поверхностью по контрасту с задним фоном, по этой границе проводится прямая для обозначения границы раздела сред; через центр окружности и точку пересечения окружности с прямой границы раздела сред вписывается прямоугольный треугольник для проведения касательной к окружности; угол смачиваемости определяется как угол между границей раздела сред и касательной к окружности.

\section{3. Результаты и обсуждение}

Качество углеродных нанотрубок принято исследовать с помощью комбинационного рассеяния света [25-27]. Многостенным нанотрубкам соответствуют высокие пики $D\left(1364 \mathrm{~cm}^{-1}\right)$ и $G\left(1580-1590 \mathrm{~cm}^{-1}\right)$, отношение которых указывает на качество МУНТ, и группа пиков в диапазоне 2500-3200 $\mathrm{cm}^{-1}$, соответствующая вторым гармоникам $D$ - и $G$-пиков, наличие которых также говорит о качестве МУНТ. Спектры многостенных УНТ от одностенных отличаются отсутствием радиальной дыхательной моды (RBM) в диапазоне $300-500 \mathrm{~cm}^{-1}$. В нашем случае спектр КРС необлученных УНТ с типичным разложением (см. рис. 1, $a$ ) соответствует характерным спектрам МУНТ.
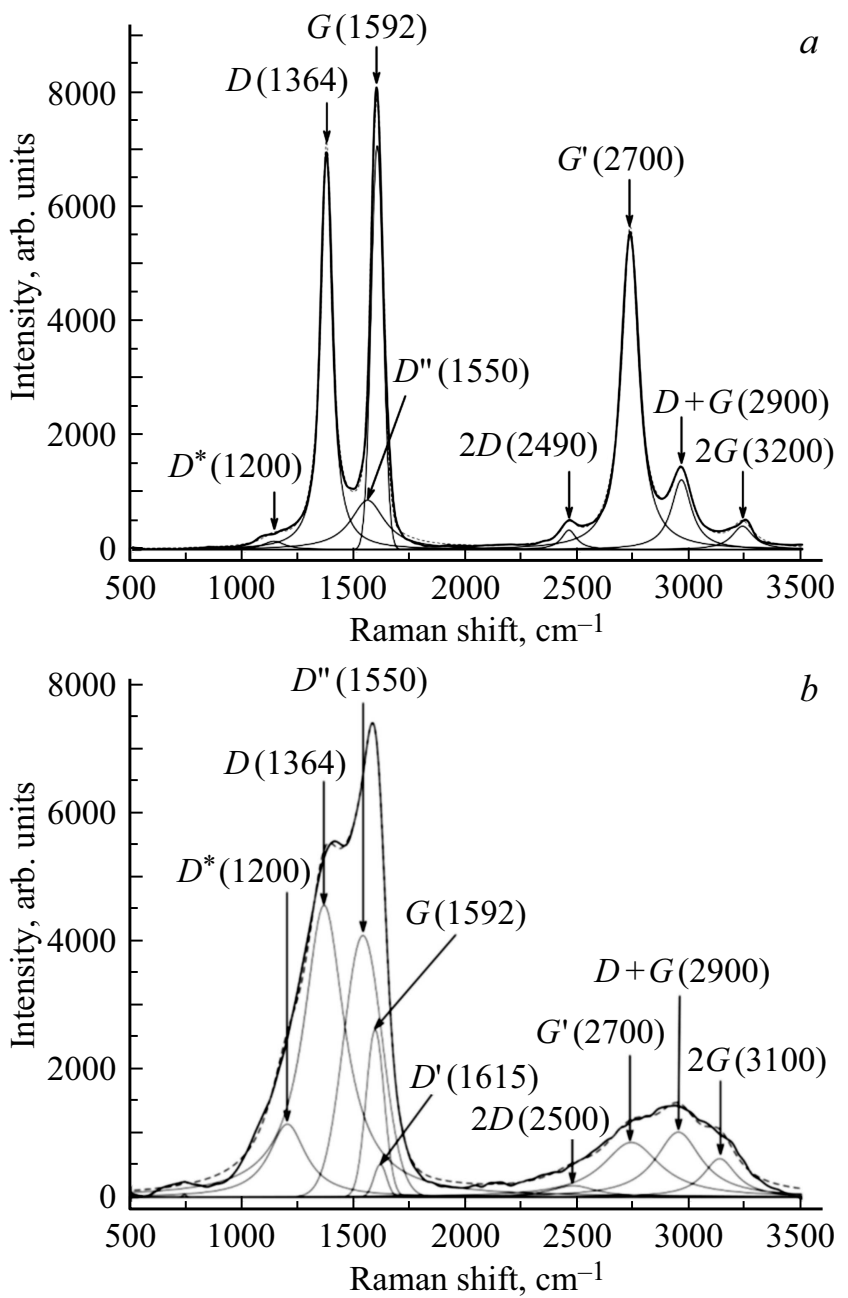

Рис. 1. Спектры КРС МУНТ и их разложение: $a-$ необлученные МУНТ ТМД, $b$ - облученные флюенсом $2 \cdot 10^{15} \mathrm{~cm}^{-2}$. 
После облучения ионами $\mathrm{Ar}^{+}$с энергией 120 кэВ видно, что произошло нарушение структуры МУНТ. В спектрах КРС наблюдается пик $D^{\prime \prime}\left(1550 \mathrm{~cm}^{-1}\right)$, интенсивность которого растет при увеличении флюенса облучения, и при флюенсе в $2 \cdot 10^{15} \mathrm{~cm}^{-2}$ он заполняет промежуток между $D$ - и $G$-пиками; также наблюдается уширение $D$-пика (см. рис. $1, b)$. Пик $D^{\prime \prime}$ объясняют как образованием кристаллитов графита [28], так и межузельными дефектами, связанными с аморфным углеродом, находящимся в состоянии $s p^{2}$-гибридизации.

При флюенсе облучения в $2 \cdot 10^{15} \mathrm{~cm}^{-2}$ наблюдается уширение $D$ - и $G$-пиков, обусловленное усилением пиков $D^{*}\left(1200 \mathrm{~cm}^{-1}\right), D^{\prime \prime}\left(1550 \mathrm{~cm}^{-1}\right)$ и $D^{\prime}\left(1615 \mathrm{~cm}^{-1}\right)$; также наблюдается резкое уменьшение интенсивности пиков второго порядка: $2 D\left(2500 \mathrm{~cm}^{-1}\right), G^{\prime}\left(2700 \mathrm{~cm}^{-1}\right)$, $D+G\left(2900 \mathrm{~cm}^{-1}\right)$ и $2 G\left(3200 \mathrm{~cm}^{-1}\right)$. Пик $D^{*}$ объясняют наличием связей $s p^{2}-s p^{3}$ на краях и изгибах углеродных нанотрубок [29]. Появляющийся при этом флюенсе $D^{\prime}$-пик связывают с наличием включений в интеркалированном графите, которые указывают на разупорядоченность структуры МУНТ [30,31]. Аналогичный эффект наблюдается в спектре образца МУНТ при флюенсе облучения $4 \cdot 10^{14} \mathrm{~cm}^{-2}$.

Анализ отношения $I_{D} / I_{G}$ указывает на увеличение степени дефектности структуры МУНТ после ионного облучения (см. рис. 2), что объясняется разрушением углеродных связей и образованием свободных связей, которые приводят к окислению МУНТ, и это коррелирует с увеличением концентрации $\mathrm{O}_{2}$ по анализу результатов РСМА.

Анализ СЭМ-изображений (см. рис. 3) показывает, что ионное облучение МУНТ приводит к увеличению среднего диаметра $\langle d\rangle$ нанотрубок (см. рис. 4), что связано с „разбуханием“ верхних слоев МУНТ. Этот эффект можно объяснить разрушением структуры МУНТ при ионном облучении [32].

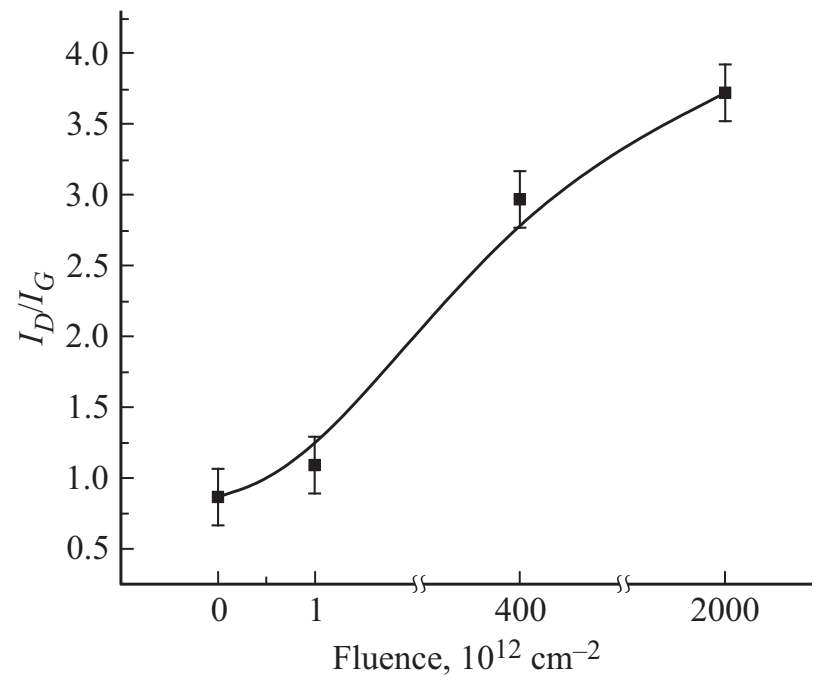

Рис. 2. Зависимость отношения интегральной интенсивности пиков $I_{D} / I_{G}$ от флюенса.
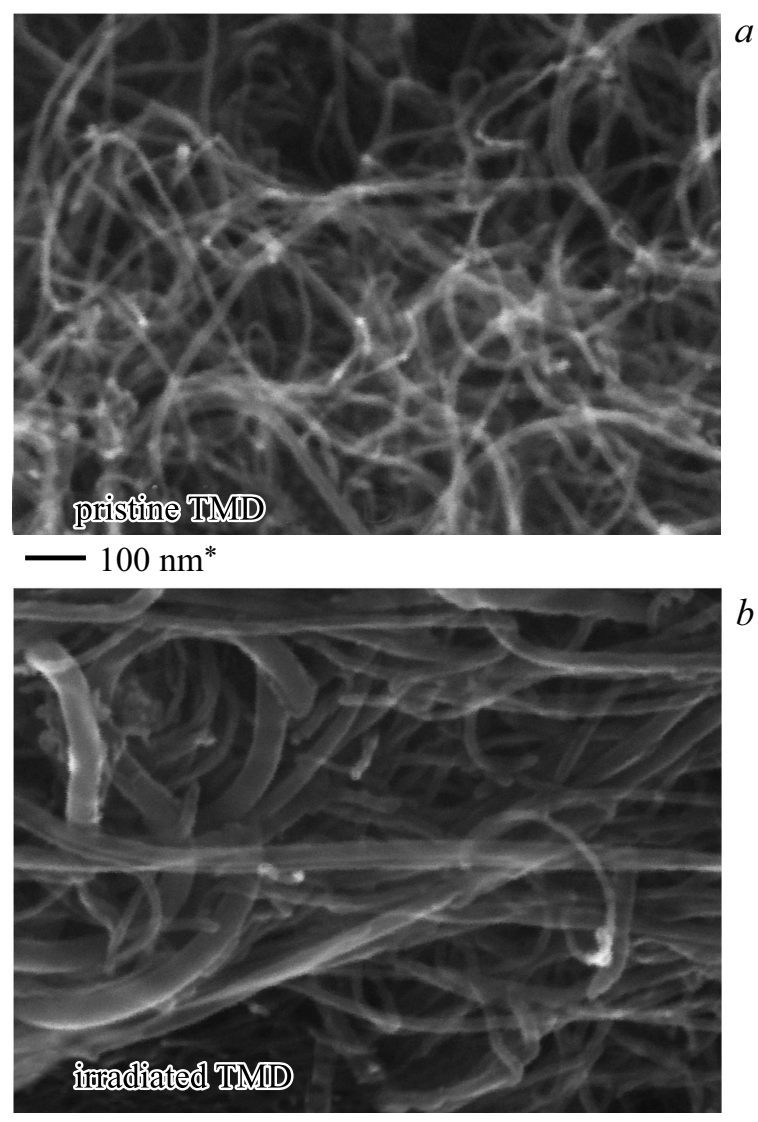

$-100 \mathrm{~nm}^{*}$

Рис. 3. СЭМ-изображения необлученного $(a)$ и облученного флюенсом $2 \cdot 10^{15} \mathrm{~cm}^{-2}(b)$ образцов МУНТ ТМД.

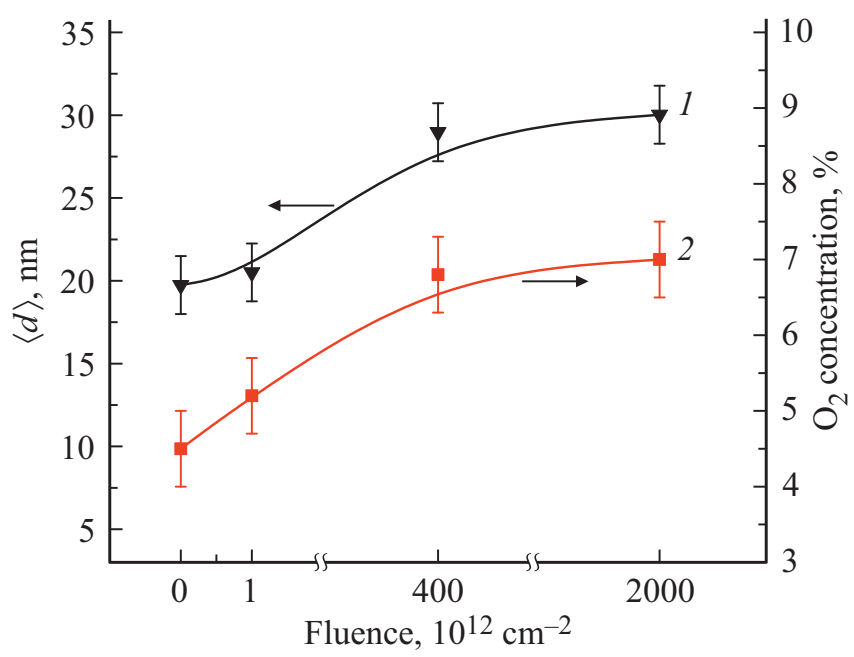

Рис. 4. Зависимости от флюенса: среднего диаметра МУНТ ТМД (1) и концентрации $\mathrm{O}_{2}$ в образцах МУНТ ТМД (2).

Также на рис. 4 приведены результаты РСМА в виде зависимости концентрации $\mathrm{O}_{2}$ от флюенса облучения. Для образцов ТМД наблюдается увеличение концентрации $\mathrm{O}_{2}$ с увеличением флюенса с последующим выходом на насыщение. Концентрация кислорода соотносится с 


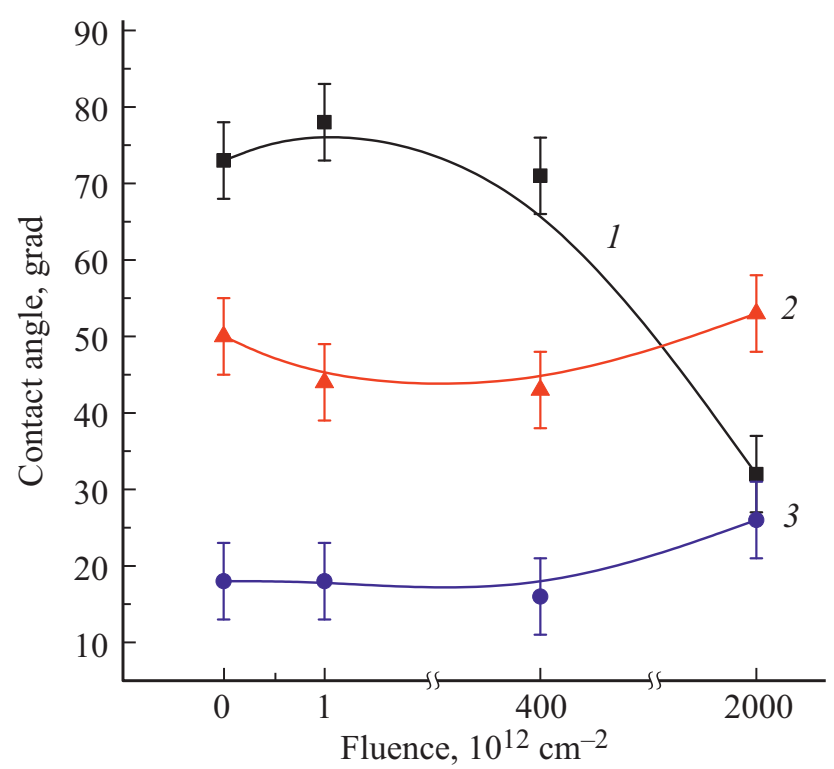

Рис. 5. Зависимость угла смачиваемости образцов МУНТ ТМД от флюенса для дистиллированной воды (1), этиленгликоля (2), циклогексана (3).

увеличением среднего диаметра МУНТ после облучения. Степень окисления поверхности УНТ сильно влияет на характер взаимодействия УНТ с жидкостями в зависимости от концентрации кислорода наблюдается переход от гидрофобности к гидрофильности [33].

Измерение угла смачиваемости образцов МУНТ ТМД после ионного облучения флюенсом $2 \cdot 10^{15} \mathrm{~cm}^{-2}$ показывает, что ионное облучение приводит к заметному уменьшению угла смачиваемости поверхности дистиллированной водой и к небольшому увеличению для этиленгликоля и циклогексана (см. рис. 5). Для случаев облучения при других флюенсах наблюдается незначительное изменение угла смачиваемости для всех тестируемых жидкостей.

Изменение угла смачиваемости при флюенсе $2 \cdot 10^{15} \mathrm{~cm}^{-2}$ коррелирует с увеличением диаметра МУНТ ТМД, увеличением концентрации $\mathrm{O}_{2}$ и увеличением степени дефектности МУНТ.

Исходя из динамики зависимости угла смачиваемости от флюенса можно сделать предположение о том, что облучение с более высоким флюенсом позволит создавать гидрофильные покрытия из МУНТ ТМД для дистиллированной воды и гидрофобные для этиленгликоля и циклогексана.

\section{4. Заключение}

Облучение ионами $\mathrm{Ar}^{+}$с энергией 120 кэВ МУНТ Таунит-МД приводит к увеличению их среднего диаметра при увеличении флюенса облучения, что подтверждается анализом СЭМ-изображений. Этот эффект ионного облучения можно объяснить образованием новых свободных связей из-за внесения дефектов в структуру поверхности МУНТ.

Анализ отношения $I_{D} / I_{G}$ из спектров КРС указывает на увеличение степени дефектности МУНТ с увеличением флюенса облучения, что приводит к увеличению концентрации $\mathrm{O}_{2}$ в образцах.

Для образцов ТМД при флюенсе облучения $2 \cdot 10^{15} \mathrm{~cm}^{-2}$ наблюдается уменьшение угла смачиваемости для дистиллированной воды и небольшое увеличение угла смачиваемости для циклогексана и этиленгликоля, при меньших флюенсах изменение смачиваемости этими жидкостями незначительное. Увеличение угла смачиваемости для дистиллированной воды находится в прямой зависимости от среднего диаметра МУНТ и концентрации $\mathrm{O}_{2}$ в образцах, что подтверждается анализом спектров комбинационного рассеяния света, СЭМ-изображениями и рентгеноспектральным микроанализом.

\section{Финансирование работы}

Работа выполнена при поддержке гранта Российского научного фонда (РНФ) № 18-72-00149.

А.П. Евсеев является стипендиатом Фонда развития теоретической физики и математики „БАЗИС“.

\section{Конфликт интересов}

Авторы заявляют, что у них нет конфликта интересов.

\section{Список литературы}

[1] S. Iijima. Nature, 354, 56 (1991).

[2] E.M. Elsehly, N.G. Chechenin, A.V. Makunin, A.A. Shemukhin, H.A. Motaweh. Rad. Phys. Chem., 146, 19 (2018).

[3] K.D. Kushkina, A.A. Shemukhin, E.A. Vorobyeva, K.A. Bukunov, A.P. Evseev, A.A. Tatarintsev, K.I. Maslakov, N.G. Chechenin, V.S. Chernysh. Nucl. Instr. Meth. B, 430, 11 (2018).

[4] V.A. Kobzev, N.G. Chechenin, K.A. Bukunov, E.A. Vorobyeva, A.V. Makunin. Materials Today: Proceedings, 5(12), 26096 (2018).

[5] V.I. Kleshch, A.A. Tonkikh, S.A. Malykhin, E.V. Redekop, A.S. Orekhov, A.L. Chuvilin, E.D. Obraztsova, A.N. Obraztsov. Appl. Phys. Lett., 109 (14), 143112 (2016).

[6] A.A. Krylov, S.G. Sazonkin, N.R. Arutyunyan, V.V. Grebenyukov, A.S. Pozharov, D.A. Dvoretskiy, E.D. Obraztsova, E.M. Dianov. J. Opt. Soc. Am. B, 33 (2), 134 (2016).

[7] A.I. Chernov, V.A. Eremina, J. Shook, A. Collins, P. Walker, P.V. Fedotov, A.A. Zakhidov, E.D. Obraztsova. Phys. Status Solidi B, 255 (1), 1700139 (2018).

[8] D.J. Liaw, N.R. Arutyunyan, W.-H. Chiang, V.A. Eremina, E.P. Kharitonova, E.D. Obraztsova. Phys. Status Solidi B, 255 (1), 1700283 (2018).

[9] F.D. Nicola, P. Castrucci, M. Scarselli, F. Nanni, I. Cacciotti, M.D. Crescenzi. Nanotechnology, 26 (14), 145701 (2015).

[10] P. Das, S. Dhal, S. Ghosh, S. Chatterjee, C.S. Rout, N. Ramgir, S. Chatterjee. Nucl. Instr. Meth. B, 413, 31 (2017). 
[11] S.H. Lu, M.H.N. Tun, Z.J. Mei, G.H. Chia, X. Lim, C. Sow. Langmuir, 25 (21), 12806 (2009).

[12] B. Bhushan, Y.C. Jung. Ultramicroscopy, 7, 1033 (2007).

[13] H. Kyakuno, M. Fukasawa, R. Ichimura, K. Matsuda, Y. Nakai, Y. Miyata, T. Saito, Y. Maniwa. J. Chem. Phys., 145, 064514 (2016).

[14] A. Ghasemi, H. Amiri, H. Zare, M. Masroor, A. Hasanzadeh, A. Beyzavi, A.R. Aref, M. Karimi, M.R. Hamblin. Microfluid. Nanofluidics, 21, 1 (2017).

[15] L. Yu, H. Hu, H.B. Wu, X.W. Lou. Adv. Mater., 29(15), 1604563 (2017).

[16] G. Wu, P. Tan, D. Wang, Z. Li, L. Peng, Y. Hu, C. Wang, W. Zhu, S. Chen, W. Chen. Sci. Rep., 7, 43676 (2017).

[17] G.K. Dimitrakakis, E. Tylianakis, G.E. Froudakis. Nano Lett., 8, 3166 (2008).

[18] J.-M. Tulliani, B. Inserra, D. Ziegel. Micromachines, 10, 232 (2019).

[19] J. Yang, Z. Zhang, X. Men, X. Xu, X. Zhu. Langmuir, 26, 10198 (2010).

[20] Y. Zhang, L. Chen, Z. Xu, Y. Li, M. Shan, L. Liu, Q. Guo, G. Chen, Z. Wang, C. Wang. Int. J. Mater. Product Technol., 45, 1 (2012).

[21] S. Baldo, V. Scuderi, L. Tripodi, A. La Magna, S.G. Leonardi, N. Donato, G. Neri, S. Filice, S. Scalese. J. Sens. Sens. Syst., 4, 25 (2015).

[22] Yu.V. Balakshin, A.A. Shemukhin, A.V. Nazarov, A.V. Kozhemiako, V.S. Chernysh. Tech. Phys., 63 (12), 1861 (2018).

[23] A.V. Kozhemiako, A.P. Evseev, Yu.V. Balakshin, A.A. Shemukhin. Semiconductors, 53 (6), 800 (2019).

[24] A.A. Shemukhin, Yu.V. Balakshin, A.P. Evseev, V.S. Chernysh. Nucl. Instr. Meth. B, 406, 507 (2017).

[25] M.S. Dresselhaus, G. Dresselhaus, R. Saito, A. Jorio. Phys. Rep., 409, 47 (2005).

[26] S. Costa, E. Borowiak-Palen, M. Kruszyńska, A. Bachmatiuk, R.J. Kaleńczuk. Mater. Sci.-Pol., 26 (2), 434 (2008).

[27] L. Bokobza, J. Zhang. eXPRESS Polym. Lett., 6(7), 601 (2012).

[28] A. Sadezky, H. Muckenhuber, H. Grothe, R. Niessner, U. Poschl. Carbon., 43, 1731 (2005).

[29] S. Vollebregt, R. Ishihara, F.D. Tichelaar, Y. Hou, C.I.M. Beenakker. Carbon, 50, 3542 (2012).

[30] J.H. Lehman, M. Terrones, E. Mansfield, K.E. Hurst, V. Meunierg. Carbon, 49, 2581 (2011).

[31] N. Chakrapani, S. Curran, B. Wei, P.M. Ajayan, A. Carrillo, R.S. Kane. J. Mater. Res., 18 (10), 2515 (2003).

[32] H. Dai, A. Javey, E. Pop, D. Mann, W. Kim, Y. Lu. NANO: Brief Rep. Rev., 1 (1), 1 (2006).

[33] M. Pavese, S. Musso, S. Bianco, M. Giorcelli, N. Pugno. J. Phys.: Condens. Matter, 20, 474206 (2008).

\section{Modification of carbon nanotubes wettability by ion irradiation}

\author{
A.I. Morkovkin ${ }^{1}$, E.A. Vorobyeva ${ }^{2}$, A.P. Evseev ${ }^{1,2}$, \\ Yu.V. Balakshin ${ }^{2,3}$, A.A. Shemukhin ${ }^{2,3}$ \\ ${ }^{1}$ Moscow State University (Faculty of Physics), \\ 119991 Moscow, Russia \\ ${ }^{2}$ Moscow State University, Skobeltsyn Institute \\ of Nuclear Physics, \\ 119991 Moscow, Russia \\ ${ }^{3}$ Center for Quantum Technologies, \\ Lomonosov Moscow State University, \\ 119991 Moscow, Russia
}

Abstract This paper presents the results of experiment to change wettability of commercial multi-walled carbon nanotubes (MWCNTs) Taunit-MD after irradiation with $120 \mathrm{keV} \mathrm{Ar}^{+}$ions with various fluences. Using the Raman spectroscopy and scanning electron microscopy, the structure of the irradiated MWCNTs was investigated and $X$-ray microanalysis was carried out. The dependences of the average diameter of the MWCNTs, the concentration of $\mathrm{O}_{2}$ in the samples, and the defectiveness of the MWCNTs on the irradiation fluence, as well as their effect on the wettability angle with distilled water, ethylene glycol and cyclohexane are considered. The possibility and prospects of using ion-beam modification methods for the controlled change of the wetting angle in order to create a hydrophobic or hydrophilic MWCNTs coating to various types of liquids are discussed.

Редактор Л.В. Шаронова 